\title{
Priming as a Means of Preventing Skill Failure Under Pressure
}

\author{
Kelly J. Ashford and Robin C. Jackson \\ Brunel University
}

\begin{abstract}
The present study examined the effectiveness of a priming paradigm in alleviating skill failure under stress. The priming intervention took the form of a scrambled sentence task. Experiment 1: Thirty-four skilled field-hockey players performed a dribbling task in low- and high-pressure situations under single task, skill-focused, and priming conditions. Results revealed a significant increase in performance time from low to high pressure. In addition, performance in the priming condition was significantly better than in the control and skill-focused conditions. Experiment 2: Thirty skilled field-hockey players completed the same dribbling task as in Experiment 1; however, in addition to the control and skill-focused conditions, participants were allocated to either a positive, neutral, or negative priming condition. Results revealed significant improvements in performance time from the skill focus to the control to the priming condition for the positive and neutral groups. For the negative group, times were significantly slower in the priming condition. Results are discussed in terms of utilizing priming in a sporting context.
\end{abstract}

Keywords: choking, skill execution, explicit monitoring, attention

Competitive environments magnify the importance of doing well and often create considerable pressure for those performing; the ability to deal with these pressures is often reflected in performance. Many performers experience suboptimal performance compared with nonpressure situations, despite having the ability to execute skills successfully (e.g., Lewis \& Linder, 1997). The term used to describe this phenomenon is choking (Baumeister, 1984), and has been defined as "the occurrence of inferior performance despite striving and incentives for superior performance" (Baumeister \& Showers, 1986, p. 361). There has been increasing interest in the underlying attentional processes of both optimal and suboptimal performance; researchers have demonstrated that the manner in which experienced performers allocate their attentional resources can have an impact on their performance (e.g., Beilock \& Carr, 2001; Beilock, Bertenthal, McCoy, \& Carr, 2004; Gray, 2004; Liao $\&$ Masters, 2002). Specifically, the literature documents that increased pressure can result in performers consciously attending to specific aspects of previously autonomous performance. Attending to a skill in an attempt to monitor and control

Kelly J. Ashford and Robin C. Jackson are with the School of Sport and Education, Brunel University, Uxbridge, Middlesex, United Kingdom. 
it can leave expert performers vulnerable to errors and more often than not, results in suboptimal performance (e.g., Anderson, 1982; Beilock, Carr, MacMahon, \& Starkes, 2002; Lewis \& Linder, 1997; Masters, 1992; Perkins-Ceccato, Passmore, \& Lee, 2003; Wulf \& Prinz, 2001). In support, Wulf, McNevin, and Shea (2001) proposed that adopting an external focus reduces ". . . active intervention into control processes ... [and] allows the more effective and natural interplay between voluntary and reflexive control processes to emerge" (p. 1152).

The term explicit monitoring (Beilock \& Carr, 2001) has been used to refer collectively to the conceptual notions of self-focus. Specifically, evidence suggests that individuals who acquire a large pool of explicit knowledge during learning are susceptible to performance decrements under pressure (Liao \& Masters, 2002). Whereas skills acquired in an environment that limits explicit knowledge results in robustness under pressure (e.g., Hardy, Mullen, \& Jones, 1996; Masters, 1992; Maxwell, Masters, \& Eves, 2000). Further, it has been suggested that skills learned and practiced in conditions that engender a high degree of self-focus would be more resilient to the detrimental effects of stress (Liao \& Masters, 2002). Research conducted by Lewis and Linder (1997) and Beilock and Carr (2001) demonstrated that learning a skill in the presence of a camera led to a stress resistant performance that eliminated the occurrence of choking. While it was argued that the findings could be confounded, in terms of whether the performers had become acclimatized to the stress associated with the presence of a camera or the self-attention that it evoked (Liao \& Masters, 2002), it can be suggested that the most important aspect of the finding was that regardless of the mechanism that brought about the change, the training environment was able to eliminate the occurrence of choking.

A significant body of research has been directed toward these issues and methods have been developed that propose to identify who is most susceptible to the internalization of attention and hence choking (e.g., Baumeister, 1984; Baumeister \& Showers, 1986; Beilock \& Carr, 2001; Beilock, Bertenthal et al., 2004; Gray, 2004; Jackson, Ashford \& Norsworthy, 2006; Lewis \& Linder, 1997; Liao \& Masters, 2002; Masters, 1992). Nonetheless, there is limited knowledge regarding strategies that may be effective in alleviating or preventing the disruptive effect conscious processing can have in athletes who have already acquired the skills necessary for their sport. Thus, the current study sought to propose a cognitive strategy that could be adopted by skilled performers to alleviate and/or prevent conscious processing from occurring, and as a consequence reduce the occurrence of choking.

There is some evidence that engaging in a concurrent secondary task while performing a motor task is effective in reducing the cognitive interference associated with skill-focused attention and can actually improve performance (e.g., Beilock, Carr, et al., 2002; Jackson et al., 2006; Maxwell et al., 2000). However, it is important that laboratory techniques can be practically adopted in a natural setting (Abernethy, 1988). From previous research (e.g., Beilock, Carr et al., 2002; Jackson et al., 2006) it is clear that, while being effective, a secondary task involving verbal responses throughout performance may not always be practical in actual competitive situations. Thus, development of a strategy that is both applicable to an ecologically valid environment and that elicits the same response as a secondary task is warranted.

Elicitation of behavioral or perceptual change has been observed in a variety of domains as a result of priming (e.g., Bargh, Chen, \& Burrows, 1996; Hull, Slone, 
Meteyer, \& Matthews, 2002); where priming refers to "the influence a stimulus has on subsequent performance of the processing system" (Baddeley, 1997, p. 352). The stimuli presented in these priming paradigms are often implicit in nature; that is, participants are not aware of the nature of the prime or its presentation (Bargh et al., 1996; Hull et al., 2002).

Priming research has consistently demonstrated activation of responses as a result of these implicit or subconscious cues (e.g., Bargh et al., 1996; Hull et al., 2002; Nelson \& Norton, 2005; Rasinski, Visser, Zagatsky, \& Rickett, 2005; Srull \& Wyer, 1979; Seinela, Hamalainen, Kovisto, \& Ruutiainen, 2002). A wide range of effects have been reported in the literature, for example, Bargh et al. (1996) and Hull et al. (2002) used a scrambled sentence task (Srull \& Wyer, 1979) in which participants were presented with a series of five-word items and were required to use four of the words to form a grammatically correct sentence. Each item contained a target word that was context specific, and which had been selected on the basis of previous research. Bargh et al. (1996) and Hull et al. (2002) demonstrated that they could decrease the speed participants walked over a set distance by giving them elderly stereotype primes. Walking speed has also been manipulated in other studies as a result of animal (e.g., cheetah) and racing driver (e.g., Michael Schumacher) primes (Aarts \& Dijksterhuis, 2002 and MaCrae et al., 1998 respectively). In both cases the activation of speed resulted in faster walking. Hull et al. (2002) also observed that they could decrease participants' reaction times on a decision making task, thus improving their performance, by giving them a success prime. Further, Srull and Wyer (1979) and Carver, Ganellen, Froming, and Chambers (1983) established that having received a hostile or kindness prime individuals perceived a neutral target with relative hostility or kindness as well as being more hostile or kind themselves.

In describing the patterns of behavioral responses to primes it has been argued that the behaviors elicited by primes are an automatic consequence of their content (Hull et al., 2002), and that the effect of priming will be greatest when the stimuli and associated responses overlap significantly (Bruce, Carson, Burton, \& Ellis, 2000). Thus, it can be suggested that primes developed to manipulate an individual's focus toward global aspects of performance would be advantageous, as they would encourage automaticity rather than the dechunking of previously automated skills into their constituent parts.

Utilizing the priming paradigm initially developed by Srull and Wyer (1979), and later adopted by Bargh et al. (1996) and Hull et al. (2002), the aim of the current study was, first, to examine the efficacy of priming as a means of enhancing motor performance and, second, to explore the potential application of priming in preventing choking. Specifically, it was hypothesized that primes associated with automaticity would enhance performance relative to control, skill-focused (Experiment 1) and negative (Experiment 2) priming conditions.

To extend the findings of previous researchers (e.g., Beilock, Carr, et al., 2002; Jackson et al., 2006), a final aim was to examine whether variations in performance could be attributed to a general decrease in the speed of motor performance and/or a decrease in technical accuracy. Beilock, Carr, et al. (2002) and Jackson et al. (2006) both adopted a soccer-dribbling task in their studies, and used time as the performance measure. While they both attempted to control accuracy by informing participants that they would repeat trials if an error in dribbling performance occurred, there was 
no evaluation of accuracy. Thus, cases may have arisen where participants remained in control of the ball but the kinematics of movement or the lateral displacement around the cones may have changed resulting in a relative increase or decrease in performance time. For example, Gray (2004) conducted a study assessing the impact of attentional allocation on hitting kinematics in baseball batters. Specifically, he recorded "wind up" and "swing duration" times in external, skill-focused and control/ single-task conditions. Gray (2004) observed that under the control and external focus conditions swing execution remained unaffected, however, the skill-focused condition caused a significant degradation of performance. Hence, he concluded that performance degradation was, in part, due to interference in the sequencing and timing of batting movement as a consequence of skill-focused attention.

\section{Experiment 1: Methods}

\section{Participants}

Initially, institutional ethical approval for the study was sought and granted. Thirtyfour skilled field-hockey players were then recruited and provided written informed consent to participate in the current study. The sample comprised 16 males (age: $M$ $=22.0$ years, $S D=2.7$ years) and 18 females (age: $M=22.1$ years, $S D=4.6$ years), currently competing in the British Universities and Colleges Sport (formerly British University Sport Association) premier and southeast conference field-hockey leagues. They reported having a mean of 10.8 years' experience ( $S D=1.8$ years).

\section{Measures}

State Anxiety. The cognitive and somatic subscales from the revised Competitive State Anxiety Inventory (CSAI-2R: Cox, Martens, \& Russell, 2003) were used to assess competitive state anxiety. Participants were asked to indicate the intensity with which they were experiencing each of the 12 items on a 4-point Likert-type scale anchored by 1 (not at all) and 4 (very much so). Cox et al. (2003) reported good construct validity and acceptable internal consistency for both cognitive $(\alpha=.83)$ and somatic anxiety subscales $(\alpha=.88)$. In addition to the CSAI-2R, participants responded to the question "How much pressure did you feel that you were under during the trials you have just completed?" on a 7-point Likert-type scale anchored by 1 (no pressure) and 7 (extreme pressure).

\section{Task}

Individuals performed a field-hockey dribbling task on a carpeted indoor surface. Participants were required to use an Indian dribble to maneuver a field-hockey ball around a 12-m slalom course, marked out by cones at 1-m intervals. A standard field-hockey stick and ball were provided. All participants were required to complete trials in all of the conditions outlined below (see Attention Conditions and Pressure Manipulation sections below).

Performance Times. The time taken to complete each trial of the experiment was recorded to the nearest millisecond using a set of Newtest PowerTimer 1.0 (Ser. No. 10187) photoelectric cells. 
Lateral Displacement. Lateral displacement was assessed by recording the maximum displacement of the ball corresponding to each cone of the task. To determine lateral displacement a reference grid was marked on the floor adjacent to each of the cones. Each zone of the reference grid was $5 \mathrm{~cm}$ wide and the distance from the midline to the first zone was $10 \mathrm{~cm}$. A digital video camera (Panasonic NVDS65B) was used to record each trial (the camera was hidden from view to prevent its presence from evoking a stress response).

Scores were calculated based on the mean maximal lateral displacement for each cone in each combination of attention and pressure conditions. In addition, $10 \%$ of trials in each condition were randomly selected and assessed by an independent rater.

\section{Attention Conditions}

Control. In the control condition, participants were simply asked to "complete the dribbling task as quickly and accurately as possible."

Skill Focus. In accordance with Baumeister (1984), in the skill-focus condition, individuals were given the instructions to "be aware of what you are doing," and "focus on the movement of your hands throughout the trial." Thus, upon hearing a tone that sounded on a 6-s variable-interval schedule, individuals could indicate verbally whether they had just pronated or supinated their hand by using the words "down" and "up," respectively.

Priming. The priming condition took the form of a scrambled sentence task (Bargh et al., 1996; Hull et al., 2002; Srull \& Wyer, 1979). Researchers have consistently demonstrated that individuals respond to subconscious cues (e.g., Bargh et al., 1996; Chen \& Bargh, 1997; Hull et al., 2002; Nelson \& Norton, 2005; Rasinski et al., 2005; Seinela et al., 2002); therefore, the aim of the priming task was to prime aspects of autonomous performance to influence participant behavior. The scrambled sentence priming task (Bargh et al., 1996; Hull et al., 2002; Srull \& Wyer, 1979) comprised 30 five-word items presented in a random order: for example, "movement the smooth was could." Before completing the trials in the priming condition, in the rest period, participants were asked to complete a grammatical task. In line with Srull and Wyer (1979), they were informed that this task was part of a different experiment; all participants agreed to participate. Participants were instructed to use four of the five words presented to them to form a grammatically correct sentence, for example, "the movement was smooth." Participants had unlimited time to complete the task. Target words were selected on the basis of criteria associated with autonomous performance: for example, "spontaneously," "balanced," and "immersed."

Pressure Manipulation. High pressure was induced by the presence of a video camera (in addition to the camera being used to measure displacement), which was operated by a confederate of the same gender as the participant. This resulted in three people being present in the laboratory during the high-pressure conditions (the participant, the first author, and the confederate). A cover story was also presented in which participants were informed that the footage of them completing the task would be used in a film about the basic skills of field hockey that the researcher was to present to the national governing body. The presence of a video camera and 
evaluation apprehension are two techniques that have been used successfully by a number of researchers to manipulate pressure (e.g., Baumeister, 1984; Beilock \& Carr, 2001; Carver \& Scheier, 1981; Liao \& Masters, 2002; Masters, 1992). Before setting up the second camera, all participants gave consent to being filmed. During the low pressure conditions, only the participant and first author were present in the laboratory.

\section{Procedure}

Having provided demographic information, participants were given three trials to familiarize themselves with the task before the experiment commenced. All trials were timed to ensure consistency across consecutive trials (Ford, Hodges, \& Williams, 2005). Before the testing phase, participants were informed that they would complete three trials in each of six conditions, that they would receive a specific set of instructions for each condition, and that each trial would be timed. To ensure that participants maintained accuracy throughout all trials and completed the course utilizing the appropriate technique, they were also informed that if any significant errors occurred, the trial would be repeated (Beilock, Carr, et al., 2002). Significant errors included knocking over a cone or losing control of the ball beyond the farthest line on the reference grid (errors occurred in approx. 5\% of trials; hence, these were repeated).

To counteract the possibility of order effects, conditions were counter-balanced with respect to pressure and attention conditions. Specifically, half of the participants completed trials in the low-pressure condition first with the other half completing trials in the high-pressure condition first. The attention conditions were counterbalanced across participants and the order for each participant was the same for the low- and high-pressure trials. To establish that the pressure manipulation was successful, the cognitive and somatic subscales of the CSAI-2R were administered following the implementation of the low/high pressure manipulation and before completion of the test trials. Finally, after both the low- and high-pressure conditions, participants completed the pressure rating question. Having completed the experimental procedure, participants were fully debriefed about the nature and purpose of the study and thanked for their participation.

\section{Data Analysis}

Data screening using standardized scores $(z \pm 3.29)$ and Mahalanobis distance test methods were conducted. Two $2 \times 3$ (Pressure $\times$ Attentional Focus) repeatedmeasures ANOVAs were conducted with repeated measures on the pressure and attentional focus factors. Mean trial completion time and mean lateral displacement served as the dependent variables.

\section{Experiment 1: Results}

\section{Data Screening}

Data screening revealed one multivariate outlier $(p<.001)$, which was subsequently removed. 


\section{Manipulation Checks}

Baseline Measures. To assess consistency across the three trials of the familiarization phase, a one-way repeated-measures ANOVA was conducted with trial number entered as the repeated factor and trial completion time serving as the dependent variable. The analysis revealed a nonsignificant effect of trial number, Wilks's $\Lambda=.95, F(2,32)=.80, p>.05, \eta_{p}^{2}=.05$ (Trial $1: M=9289$ $\mathrm{ms}, S D=1798 \mathrm{~ms}$; Trial 2: $M=9076 \mathrm{~ms}, S D=1897 \mathrm{~ms}$; Trial 3: $M=9161 \mathrm{~ms}$; $S D=2027 \mathrm{~ms})$.

Anxiety Intensity. Data obtained from the cognitive and somatic anxiety subscales were entered into a $2 \times 2$ (Pressure $\times$ Anxiety) repeated measures MANOVA. Results revealed a significant main effect for pressure, Wilks's $\Lambda=.23, F(4,29)=24.11$, $p<.01, \eta_{\mathrm{p}}^{2}=.77$. Follow-up univariate results revealed significant increases in cognitive anxiety intensity $F(1,32)=39.55, p<.01, \eta_{\mathrm{p}}{ }^{2}=.76$, and somatic anxiety intensity, $F(1,32)=5.66, p<.05, \eta_{\mathrm{p}}{ }^{2}=.16$ from the low- to high- pressure condition (see Table 1 for descriptive statistics).

\section{Table 1 Descriptive Statistics for Anxiety Intensity and Perceived Pressure Across Low and High Pressure Conditions}

\begin{tabular}{|c|c|c|c|c|c|}
\hline & \multirow[b]{2}{*}{ Pressure } & \multicolumn{2}{|c|}{$\begin{array}{c}\text { Experiment } \\
1 \\
\end{array}$} & \multicolumn{2}{|c|}{$\begin{array}{c}\text { Experiment } \\
2\end{array}$} \\
\hline & & $M$ & $S D$ & $M$ & $S D$ \\
\hline \multirow[t]{2}{*}{ Cognitive Anxiety } & Low & 11.13 & 3.53 & 11.09 & 3.61 \\
\hline & High & 18.59 & 5.18 & 18.60 & 5.51 \\
\hline \multirow[t]{2}{*}{ Somatic Anxiety } & Low & 12.35 & 2.98 & 12.61 & 3.12 \\
\hline & High & 13.78 & 4.19 & 13.81 & 4.33 \\
\hline \multirow[t]{2}{*}{ Perceived Pressure } & Low & 3.06 & 1.35 & 3.03 & 1.38 \\
\hline & High & 4.56 & 1.42 & 3.60 & 1.43 \\
\hline
\end{tabular}

Pressure Ratings. Results of a paired samples $t$ test revealed a significant difference in perceived pressure, $t(33)=-3.38, p<.001,95 \% \mathrm{CI}:-.80$ to -.20 , between the experimental conditions with mean scores indicating that perceived pressure increased from the low-pressure $(M=3.06, S D=1.35)$ to the high-pressure $(M=4.56, S D=1.42)$ condition. Reflecting upon these mean scores, it should be noted that while perceived pressure did increase from the low- to high- pressure conditions, it only reached a moderate level.

\section{Inter-Rater Reliability}

To establish objectivity, two individuals assessed the lateral displacement of the hockey ball in a random selection of trials (10\%) from each condition. Calculation of Pearson's correlation coefficient indicated the scores of the judges were highly correlated $r=.83, p<.001$ (Judge A: $M=36.30 \mathrm{~cm}, S D=5.90 \mathrm{~cm}$; Judge B: $M$ $=37.42 \mathrm{~cm}, S D=6.30 \mathrm{~cm})$. 


\section{Performance Times}

Results of a repeated-measures ANOVA revealed a nonsignificant pressure $\times$ attentional focus interaction, $F(2,31)=1.28, p>.05, \eta_{\mathrm{p}}{ }^{2}=.07$. Significant main effects for pressure, $F(1,32)=31.82, p<.001, \eta_{\mathrm{p}}{ }^{2}=.50$, and attentional focus, $F(2,31)$ $=43.67, p<.001, \eta_{\mathrm{p}}^{2}=.74$ were observed (see Figure 1). Pairwise comparisons indicated that performance was significantly faster in the priming condition than in the control condition, and significantly faster in the control condition than in the skill-focus condition. In addition, performance was significantly faster in the low-pressure than in the high-pressure condition (see Figure 1).

Finally, a series of paired samples $t$ tests (using Bonferroni adjusted alpha levels set at $p<.0125$ ) were conducted to examine the a priori hypothesis that priming elicits a positive performance effect under pressure. Results revealed there was no significant difference between the low-pressure control and the high-pressure prime condition, $t(32)=-2.12, p>.0125,95 \%$ CI: -518.99 to -10.36 , or between the low-pressure skill-focus and high-pressure control conditions, $t(32)=-1.40, p>$ $.0125,95 \%$ CI: -419.09 to 90.95 . In addition, a significant difference was observed between the low-pressure control and the high-pressure control conditions, $t(32)=$ $-4.86, p<.0125,95 \% \mathrm{CI}:-922.22$ to -377.83 , with mean scores indicating faster performance under low pressure. Further, a significant difference was observed between the high-pressure control and the high-pressure prime conditions, $t(32)$ $=3.88, p<.0125,95 \%$ CI: 182.78 to 587.92 , with mean scores indicating faster performance in the priming condition (see Figure 1).

\section{Lateral Displacement}

Results of the repeated-measures ANOVA revealed a nonsignificant pressure $\mathrm{x}$ attentional focus interaction, $F(1,31)=1.22, p>.05, \eta_{\mathrm{p}}{ }^{2}=.07$, and a nonsignificant main effect of pressure, $F(1,32)=.24, p>.05, \eta_{\mathrm{p}}{ }^{\mathrm{p}}=.01$. However, the main effect of attentional focus was significant, $F(2,31)=8.66, p<.01, \eta_{p}{ }^{2}=.36$, with pairwise comparisons indicating that lateral displacement was significantly lower in the priming condition than in both the control condition and the skill-focused condition. No significant difference was observed between the control and skillfocused conditions (see Figure 1).

\section{Experiment 1: Discussion}

The effectiveness of priming has been demonstrated in many domains (e.g., Hull et al., 2002; Seinela et al., 2002) and the current study provides the first indication that it can also have a positive impact in the domain of sport. Interestingly, the results revealed that the priming manipulation elicited a similar response to the dual-task methods used in previous studies (e.g., Beilock, Carr, et al., 2002; Jackson et al., 2006), suggesting that, as intended, attention was directed away from the mechanics of movement execution, thus preventing conscious processing. Moreover, results indicated that priming was effective in alleviating the impact of performance pressure, with performance in the high-pressure prime condition being better than in the high-pressure control condition and equal to the low-pressure control condition. In addition, in support of the priming method, performance in the 

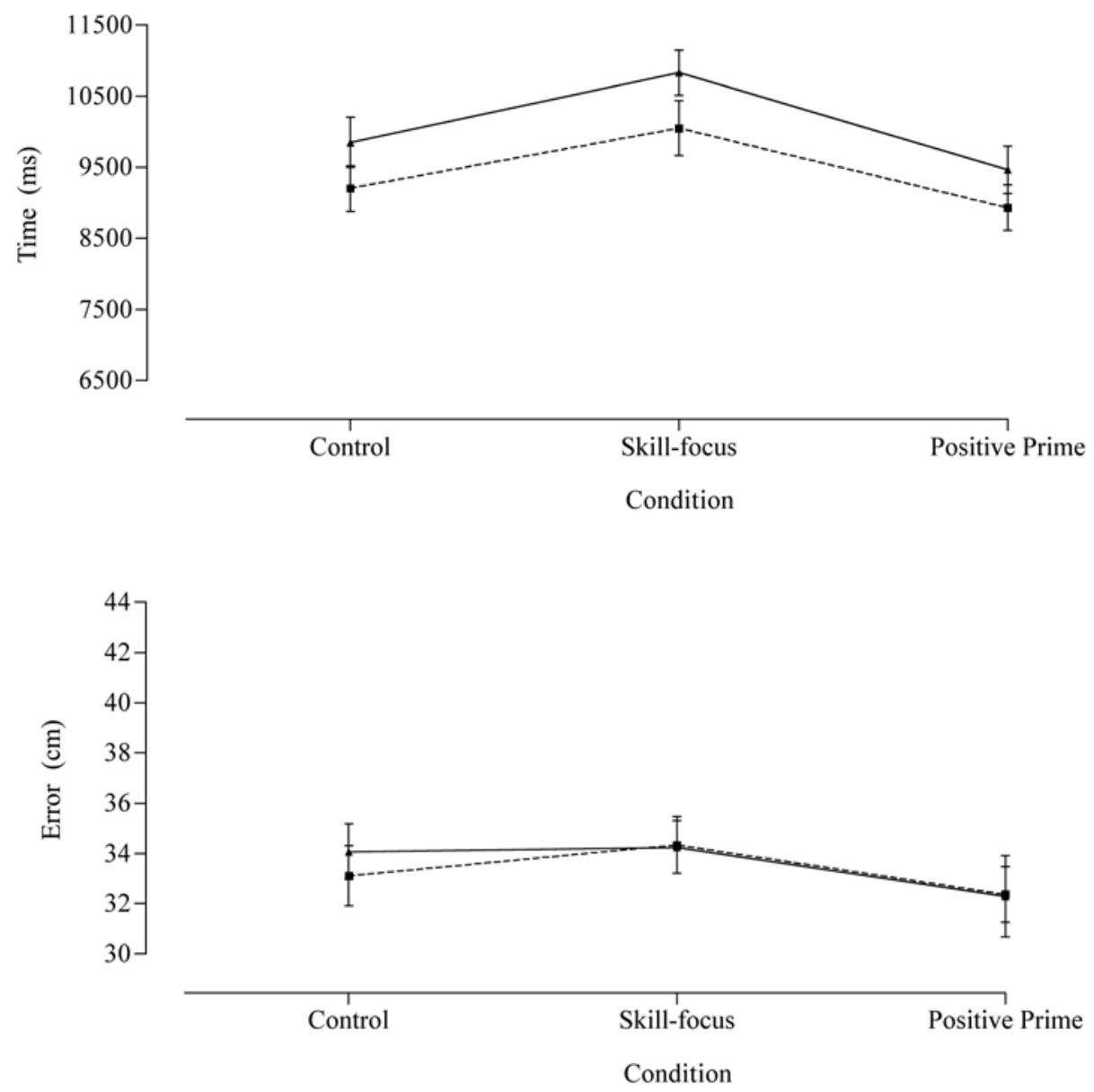

Figure 1 - Mean $( \pm S E)$ dribbling times and lateral displacement in the control, priming, and skill -focused conditions performed under low and high pressure (Experiment 1).

high-pressure priming condition was significantly better than in the high-pressure control condition. Further, results supported the findings of previous work (e.g., Beilock \& Carr, 2001; Jackson et al., 2006) highlighting that under pressure (i.e., high-pressure control) individuals are likely to direct their attention toward aspects of proceduralized skills; thus, performance in this condition was comparable with that in the low-pressure skill-focus condition. 
In line with Bruce et al. (2000), it can be suggested that the priming condition was effective because there was significant contextual overlap between the prime, the activity, and the required response. However, it must be recognized that a limitation regarding the nature of the priming task can be identified. Rather than improved performance being a consequence of the content of the prime, the facilitative effect elicited could have been a product of completing a cognitive task; that is, by occupying working memory before completion of the primary task, rumination about previous trials is minimized and attention to the skill during completion of subsequent trials is attenuated owing to rumination (consciously or unconsciously) on the previous task. During this process, the residual capacity of working memory is reduced thus decreasing the amount of available resource for explicit monitoring. To examine this further, a second experiment was conducted in which we sought to manipulate the content of the prime by randomly allocating participants to a positive, negative or neutral priming group.

\section{Experiment 2}

\section{Participants and Measures}

Initially, institutional ethical approval for the study was sought and granted. Thirty skilled field-hockey players were then recruited and provided written informed consent to participate. The sample comprised 16 males (age: $M=21.0$ years, $S D$ $=3.9$ years) and 14 females (age: $M=22.0, S D=7.9$ ), currently competing in the British Universities and Colleges Sport (formerly British University Sport Association) premier and southeast conference field-hockey leagues. They reported having a mean of 9.3 years of experience ( $S D=3.2$ years).

All measures and the procedure adopted in Experiment 1 were replicated in Experiment 2, the only variation being in the priming condition. For Experiment 2 participants were allocated to a positive, negative or neutral group in the priming condition.

\section{Priming Condition}

The task in this condition took the same form as that presented in Experiment 1. The variation in the task can be observed at the target word level. Specifically, participants were allocated to either the positive, negative or neutral priming groups. Target words for each group were selected on the basis of their association with autonomous performance (as in Experiment 1) (e.g., spontaneously, balanced, immersed), disrupted performance (e.g., breakdown, poor, slow) and neutral words unrelated to the present context (e.g., table, green, sky), respectively. Examples of sentences used for the latter two groups respectively, were as follows: "truck arrived in the breakdown" and "square round the is world."

\section{Data Analysis}

Data screening using standardized scores $(z \pm 3.29)$ and Mahalanobis distance test methods were conducted. Two $3 \times 3 \times 2$ (Priming Group $\times$ Attentional Focus $\times$ Pressure) mixed factor ANOVAs were conducted with repeated measures on the 
pressure and attentional focus factors, and priming group serving as the betweensubjects factor. Mean trial completion time and mean lateral displacement served as the dependent variables.

\section{Experiment 2: Results}

\section{Data Screening}

Data screening revealed no univariate or multivariate outliers.

\section{Manipulation Checks}

Baseline Measures. A $3 \times 3$ (Priming Group $\times$ Baseline Trial) repeated-measures ANOVA was conducted to assess consistency across trials during the familiarization stage; trial number represented the repeated measure and trial completion time served as the dependent variable. Results revealed a nonsignificant interaction, Wilks's $\Lambda=.74, F(4,52)=2.13, p>.05, \eta_{p}{ }^{2}=.09$, and nonsignificant main effects for trial number, Wilks's $\Lambda=.93, F(2,26)=.97, p>.05, \eta_{\mathrm{p}}{ }^{2}=.07$ (Trial 1: $M=$ $9273 \mathrm{~ms}, S D=1851 \mathrm{~ms}$; Trial 2: $M=9035 \mathrm{~ms}, S D=1999 \mathrm{~ms}$; Trial 3: $M=9098$ $\mathrm{ms}, S D=2088 \mathrm{~ms}$ ) and priming group, $F(2,27)=.25, p>.05, \eta_{\mathrm{p}}{ }^{2}=.02$.

Anxiety Intensity. Results from a $3 \times 2$ (Priming Group $\times$ Pressure) repeated measures MANOVA examining cognitive and somatic anxiety, revealed a nonsignificant interaction, Wilks's $\Lambda=.97, F(4,52)=.22, p>.05, \eta_{\mathrm{p}}{ }^{2}=.02$, while the main effect for pressure was significant, Wilks's $\Lambda=.26, F(2,26)=37.21, p<$ $.01, \eta_{\mathrm{p}}{ }^{2}=.74$. Results of the univariate analyses revealed significant increases in both cognitive anxiety intensity $F(1,27)=76.31, p<.01, \eta_{\mathrm{p}}{ }^{2}=.74$, and somatic anxiety intensity, $F(1,27)=3.56, p=.07, \eta_{\mathrm{p}}^{2}=.12$ from the low- to high-pressure condition (see Table 1).

Pressure Ratings. Results of a paired samples $t$ test revealed a significant difference in perceived pressure, $t(29)=-3.62, p<.001,95 \% \mathrm{CI}:-.89$ to -.25 , with mean scores indicating that perceived pressure increased from the low-pressure $(M$ $=3.03, S D=1.38)$ to the high-pressure $(M=3.60, S D=1.43)$ condition.

\section{Performance Times and Lateral Displacement}

Mixed-factor ANOVA results for trial completion time revealed a significant priming group $\times$ condition interaction, Wilks's $\Lambda=.40, F(4,52)=7.62, p<.001, \eta_{p}{ }^{2}=.37$. Analysis of simple effects revealed that completion times became progressively faster from the skill focus to the control to the priming condition for both the positive and neutral prime groups ( $p<.01$ and $p<.05$ respectively). For the negative prime group, the significant effect of condition $(p<.05)$ reflected times that were slower in the priming condition compared with the control condition, with no difference evident between times in the skill-focus and negative priming condition (see Figure 2). In addition, the significant main effect for pressure indicated that performance was slower under high pressure, Wilks's $\Lambda=.52, F(1,27)=24.57, p<.001, \eta_{\mathrm{p}}{ }^{2}$ $=.47$ (see Figure 2). 

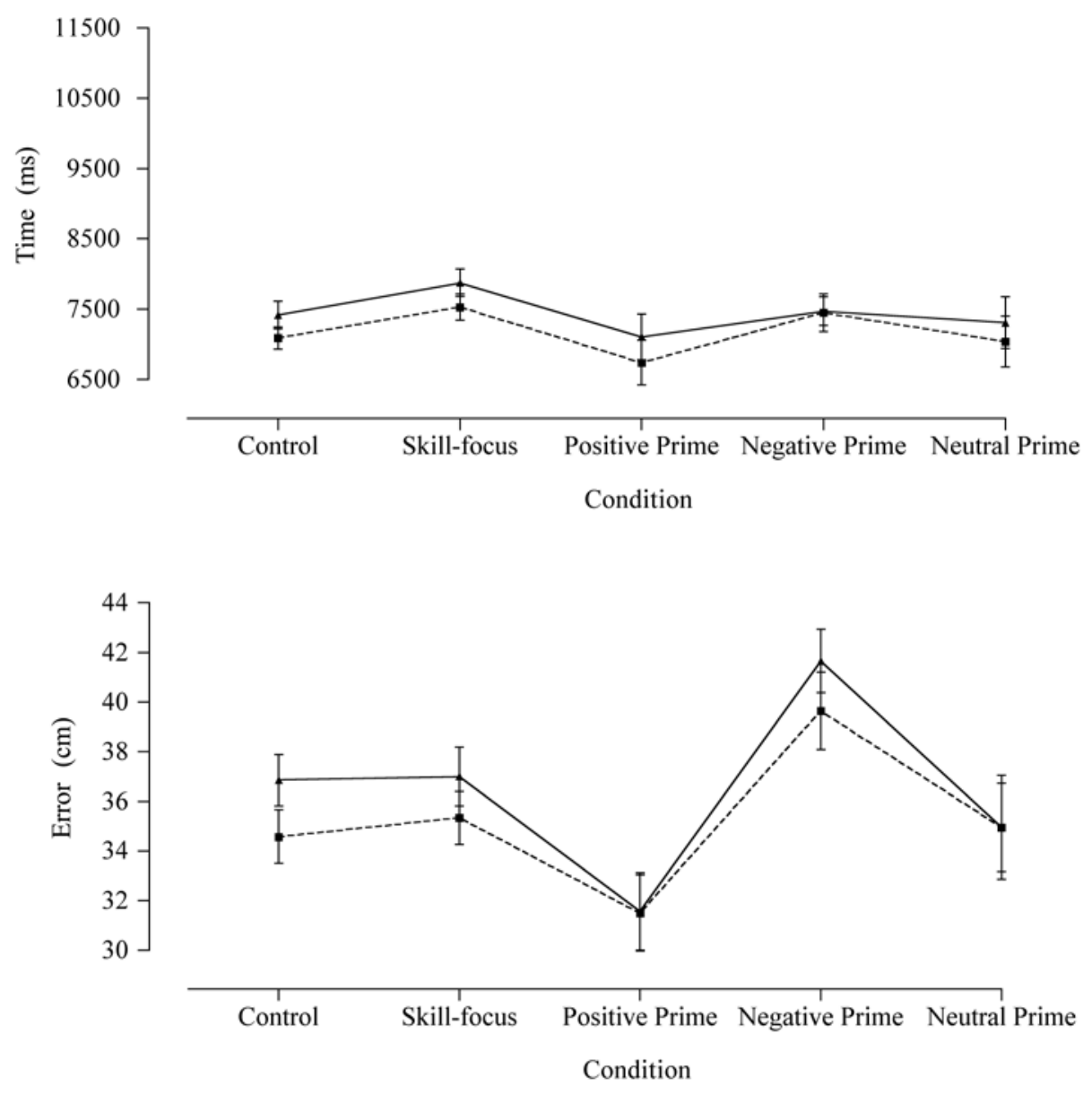

--*-- Low Pressure $\longrightarrow$ High Pressure

Figure 2 - Mean $( \pm S E)$ dribbling times and lateral displacement in the control, priming, and skill -focused conditions performed under low and high pressure (Experiment 2).

With regard to lateral displacement, the only significant difference emanating from the omnibus ANOVA was for the main effect of pressure, Wilks's $\Lambda=.71$, $F(1,26)=10.61, p<.05, \eta_{\mathrm{p}}{ }^{2}=.29$, with mean results indicating greater lateral displacement under high pressure. However, as seen in Figure 2, lateral displacement in the negative prime group was a lot greater than the other two prime groups. To explore this potential difference between groups, a $3 \times 2$ (Group $\times$ Pressure) 
repeated-measures ANOVA was conducted. The analysis revealed a significant between-group effect $F(2,26)=9.38, p<.01, \eta_{\mathrm{p}}^{2}=.42$. Post hoc analyses revealed greater lateral displacement in the negative prime group compared with the control and positive prime group.

\section{Experiment 2: Discussion}

Results were consistent with those presented in Experiment 1, supporting the effectiveness of priming in the sporting domain. In addition, they support the increasing body of literature highlighting the deleterious effects of skill-focused attention (e.g., Beilock, Carr, et al., 2002), and the positive impact of directing attention away from the mechanics of movement execution (e.g., Beilock, Carr, et al., 2002; Jackson et al., 2006).

In line with Bruce et al. (2000), it can be suggested that the content of the prime to which participants were exposed was responsible for the resultant performance in the priming condition. Specifically, rather than the positive prime having a facilitative impact based simply on its cognitive nature, it can be suggested that the ensuing performance, which was faster relative to the control and skill-focused condition, was a product of the content of the priming intervention. In support, results also show that those participants receiving negative primes were significantly slower with greater lateral displacement in the priming condition compared with the control condition with times in the priming condition approaching (and not significantly different from) those obtained in the skill-focused condition.

\section{General Discussion}

The primary aims of the current study were to examine the efficacy of priming as a means of enhancing motor performance and, second, to discover whether the utilization of a priming paradigm presented before performance could alleviate the paradoxical performance effect of choking. Previous research has identified that dual-task methods are effective in maintaining an appropriate level of attentional focus so that, under pressure, performance returns to a level akin with a low-pressure control (e.g., Beilock, Carr, et al., 2002; Jackson et al., 2006; Maxwell et al., 2000). Likewise, it has been observed that performance under skill-focused conditions is analogous to that observed during choking (Beilock, Carr, et al., 2002; Jackson et al., 2006). Hence, it was anticipated that the priming method adopted herein would influence the motor processing system resulting in an attentional focus and behavioral response that was consistent with the content of the prime.

Examination of performance times across the three attention conditions revealed significant performance decrements from the control condition to the skillfocused condition while performance in the priming condition was consistent with the content of the prime (i.e., positive primes resulted in improved performance and negative primes resulted in poorer performance compared with the control condition). Decrements in performance could also be observed from the low- to high-pressure condition. These findings lend support to the contentions that attention allocation plays a mediating role in performance degradation (e.g., Anderson, 1982; Baumeister, 1984; Beilock, Carr, et al., 2002; Carver \& Scheier, 1981; 
Gray, 2004). In particular, the results highlight the negative impact of attending to proceduralized components of a skill (whether triggered consciously or unconsciously), and the benefits of an intervention that primes participants to display features of automated performance under both low and high pressure. The latter may be particularly relevant for individuals who are susceptible to choking as the intervention has the potential to promote characteristics of skilled performance that are also retained under pressure.

The purpose of analyzing the displacement of the hockey ball was to establish whether performance decrements that occurred could be attributed to a general decline in the speed of execution or to a decrease in accuracy (hence greater ball displacement over the slalom course). In support of Gray (2004), the current study demonstrated that generally, there was a decrement in the time and accuracy of the dribbling task across the attention conditions, with it being most prevalent in the skill-focused condition and negative priming conditions (see Figures 1 and 2). Specifically, data were consistent with the view that the skill-focused/ negative priming conditions, and to a lesser extent the control condition, caused participants to focus their attention internally toward aspects of performance (e.g., Anderson, 1982; Baumeister, 1984; Beilock et al., 2004; Beilock, Carr, et al., 2002; Fitts \& Posner, 1967; Masters, 1992; Wulf \& Prinz, 2001). It has been argued that this process initiates the dechunking of the motor programs being used for the task, thus causing individuals to regress back to controlling components of skill in a stepby-step fashion. Research has consistently demonstrated that for skilled athletes, processing motor information in this declarative manner is detrimental to both the speed and accuracy of motor task execution and reflects the processing undertaken during an instance of choking (Anderson, 1982; Beilock, Carr, et al., 2002; Fitts \& Posner, 1967; Gray, 2004, Masters, 1992). Conversely, for novice athletes, this monitoring process is necessary in order for improvements in skills to take place.

From an affective perspective, it has been demonstrated that pleasant and unpleasant emotional cues have a differential impact on subsequent performance accuracy (Coombes, Janelle, \& Duley, 2005). Specifically, Coombes et al., (2005) demonstrated an increase/decrease in accuracy during a tracing task following the presentation of relative affective stimuli. Therefore, it could be suggested that as well as the contextual overlap between the prime and the task, relative temporal positive/negative affective states were elicited which contributed to the mode of processing occurring during performance. While a positive affective state may stimulate autonomous processing, a negative affective state may encourage engagement in conscious control. Thus, not all of the negative primes explicitly encouraged skill focus in the corresponding way that the positive primes encouraged features of automaticity. Determining whether the benefits of the positive primes and detrimental effects of the negative primes resulted directly from the content of the prime or indirectly via changes in affective states resulting from the primes is a logical extension of the present research.

Observing the difference between the control and priming conditions in both pressure conditions raises an interesting question: Why should it be that performance under the positive/negative priming condition was better/worse than the control condition? Similar results have been reported by researchers using a dual-task condition (tone/ word monitoring) in studies of soccer dribbling (Beilock, Carr, et al., 2002; Jackson et al., 2006) and golf putting (Beilock, Wierenga, et al., 2002). Jackson et al. (2006) 
speculated that this might be caused by attenuation of residual explicit monitoring present under control/single task conditions. Thus, it can be suggested that the positive priming intervention eliminated or significantly reduced the degree of residual explicit monitoring taking place, while the negative priming condition exacerbated the monitoring. Encouragingly, the present findings illustrate the efficacy of a priming paradigm under both low- and high-pressure conditions; however, it should be noted that measures of attention were not taken. An important next step is to substantiate our assertion that the priming effects have differential effects with respect to attentional processes. One way in which this might be done is to use a probe reaction time task to assess the attentional demands under different prime conditions. If our assertion is correct, then positive primes aimed at promoting automaticity should be associated with faster probe reaction times than those associated with negative primes.

While the results of the current study illustrate the efficacy of priming in motor behavior, the process was not necessarily implicit. Even though the sentence scrambling task was introduced as a separate experiment, participants may have been able to discern a link between the two. Nonetheless, in line with previous research (e.g., Baumeister, 1984) the effect of directing attention to the outcome of performance rather than the process resulted in improved performance. However, it remains necessary to further explore and establish the validity and reliability of the technique in both laboratory and applied contexts. Until this time, in accordance with priming in other domains (e.g., Bargh et al., 1996; Bruce et al., 2000; Hull et al., 2002), it is suggested that presenting contextually comparable primes will likely elicit the largest priming effect.

The findings provide further support for research on performance decrements resulting from self- or skill-focus (Beilock et al., 2004; Beilock, Carr, et al., 2002; Ford et al., 2005; Gray 2004; Jackson et al., 2006; Wulf \& Prinz, 2001), and provide preliminary support for the effectiveness of a priming intervention (e.g., Hull et al., 2002). However, the results raise a number of practical and theoretical issues that warrant additional investigation.

With regard to performance times, it can be noted that these were slower in Experiment 1 (see Figures 1 and 2). While all participants were recruited from the same population and hence, in terms of their level of participation and expertise, were a homogeneous sample, the male to female participant ratio was different for the two experiments. Experiment 1 contained a higher proportion of female participants who tended to be slightly slower than their male counterparts. It is also possible that there was a difference in the emphasis placed on speed and accuracy by the participants in each experiment. While the instructions to participants were consistent across the two experiments, the potential for different interpretations in participants who know one another is readily apparent.

From both a theoretical and practical perspective, it is of interest to know whether a shortened priming task would have the same positive impact on performance. This would enhance its utility in a practical sporting context, for example, by opening up the possibility that interventions could be targeted during short breaks in competition as well as before performance. Positive findings with regard to the utilization of shortened tasks have been reported; Bry, Meyer, Oberlé, and Gherson (2009) reported an improvement in relay change-over speed in beginner athletes after exposure to a 16-item sentence scrambling task while Nelson and Norton (2005) elicited helping behavior with 10 items. Further, Srull and Wyer 
compared 30 and 60 items; although the longer task resulted in a stronger effect, both tasks resulted in significant behavioral changes. Related to this issue, it is also of interest to know how long the positive impact of the priming intervention lasts. Sporting events vary greatly in length from a matter of seconds or minutes (e.g., weightlifting, ski racing) to hours (e.g., a game of field-hockey or soccer). Thus, it would be beneficial to establish whether the relatively short priming intervention adopted herein produces a transient effect (beneficial for the short duration sports) or a longer-lasting effect that might also be beneficial for sports of longer duration. Encouragingly, the durability of priming effects has been investigated by Srull and Wyer (1979) and Nelson and Norton (2005), who found that hostility toward another being and willingness to do volunteer work was activate for up to $1 \mathrm{hr}$ and three months postexposure respectively.

In terms of its application, the sentence-scrambling task could be easily integrated into a performer's precompetition routine, or adapted to use during short breaks in play (e.g., reciting sentences like those used in the positive prime condition), thereby offering an alternative or complementary psychological intervention for those individuals experiencing performance decrements. However, assessment of the latter and other methods of presenting the primes (e.g., a video containing subliminal primes or flashcards), that have the potential to engender a similar performance effect is warranted. In addition, researchers in the priming domain have used a limited array of motor skills to date, including walking (e.g., Aarts \& Dijksterhuis, 2002; Bargh et al., 1996), relay baton changeover speed (Bry, Meyer, Oberlé, \& Gherson, 2009), and golf-putting (Stone, Lynch, Sjomeling, \& Darley, 1999). Thus, examining the extent to which priming effects generalize to other motor skills is warranted.

Further, while it may be argued that the dribbling skill was taken out of context, it can also be argued that the dribbling task reflected a type of skill-based drill that individuals may undertake during a training session. Owing to the nature of the experimental setting (i.e., in the laboratory) the ecological validity of the research can be questioned on several fronts, including the nature of the task and the pressure manipulation. Clearly, it is important to establish that any psychological phenomenon can be observed, and that new techniques are effective, in a controlled setting before attempting to apply them "in the field" (Tipper \& Weaver, 1998). Accordingly, establishing the reliability and robustness of the priming effect under controlled conditions is an important and logical next step. A secondary step would be to assess the concept of self-attention and hopefully confirm the effectiveness of strategies within ecologically valid settings. The findings from such research would provide empirical support for the findings of the present research, while also providing practitioners and athletes with beneficial information regarding the paradoxical performance effect of choking and the possible facilitating effects of priming.

Finally, while the pressure manipulation used in the present study led to heightened anxiety and perceptions of pressure, the impact on performance was less marked. It can be suggested that the extent to which the camera triggered private, covert evaluations of standards and expectancies was inferior compared with what is encountered in a real competitive situation. In support, Mullen and Hardy (2000) suggest that a video camera primarily targets social or public aspects of the self, which would appear relatively unrelated to internal states. Thus, as highlighted 
above, once the effectiveness of an intervention is established within a laboratory setting, it is imperative that it is assessed within a competitive environment to establish its robustness in true situations of heightened pressure.

In conclusion, the current study took steps toward establishing the efficacy of priming individuals with autonomous words to elicit enhanced motor skill performance. Results revealed that the positive priming intervention was effective in enhancing performance when compared with a control condition, while negative priming had deleterious effects. This performance response was attributed to the attenuation/augmentation of residual explicit monitoring, respectively. In addition, and in line with a growing body of research, the present findings reinforce the proposition that attentional allocation has a significant impact on performance. In light of the present findings, developing a short-duration positive priming intervention that engenders similar effects as those demonstrated herein seems a logical next step. Further, examination of the underlying mechanisms associated with priming interventions should aid the design of ecologically valid interventions that help to prevent performance failure under pressure.

\section{References}

Aarts, H., \& Dijksterhuis, A. (2002). Category activation effects in judgment and behavior: The moderating role of perceived comparability. The British Journal of Social Psychology, 41, 123-138.

Abernethy, B. (1988). Dual-task methodology and motor skills research: Some application and methodological constraints. Journal of Human Movement Studies, 14, 101-132.

Anderson, J.R. (1982). Acquisition of a cognitive skill. Psychological Review, 89, 369-406.

Baddeley, A.D. (1997). Human Memory: Theory and practice. Hove, England: Psychology Press.

Bargh, J.A., Chen, M., \& Burrows, L. (1996). Automaticity of social behavior: Direct effects of trait construct and stereotype activation on action. Journal of Personality and Social Psychology, 71, 230-244.

Baumeister, R.F. (1984). Choking under pressure: Self-consciousness and paradoxical effects of incentives on skilful performance. Journal of Personality and Social Psychology, 46, 610-620.

Baumeister, R.F., \& Showers, C.J. (1986). A review of paradoxical performance effects: Choking under pressure in sports and mental tests. European Journal of Social Psychology, 16, 361-383.

Beilock, S.L., Bertenthal, B.I., McCoy, A.M., \& Carr, T.H. (2004). Haste does not always make waste: Expertise, direction of attention, and speed versus accuracy in performing sensorimotor skills. Psychonomic Bulletin \& Review, 11, 373-379.

Beilock, S.L., \& Carr, T.H. (2001). On the fragility of skilled performance: What governs choking under pressure? Journal of Experimental Psychology. General, 130, 701-725.

Beilock, S.L., Carr, T.H., MacMahon, C., \& Starkes, J.L. (2002). When paying attention becomes counterproductive: Impact of divided attention versus skill-focused attention on novice and experimental performance of sensorimotor skills. Journal of Experimental Psychology. Applied, 8, 6-16.

Beilock, S.L., Wierenga, S.A., \& Carr, T.H. (2002). Expertise, attention, and memory in sensorimotor skill execution: Impact of novel task constraints on dual-task performance and episodic memory. The Quarterly Journal of Experimental Psychology, 55A, 1211-1240.

Bruce, V., Carson, D., Burton, M.A., \& Ellis, A.W. (2000). Perceptual priming is not a necessary consequences of semantic classification of pictures. The Quarterly Journal of Experimental Psychology, 53A, 289-323. 
Bry, C., Meyer, T., Oberlé, D., \& Gherson, T. (2009). Effect of priming cooperation or individualism on a collective and interdependent task: Changeover speed in the $4 \mathrm{x} 100$-meter relay race. Journal of Sport \& Exercise Psychology, 31, 380-389.

Carver, C.S., \& Scheier, M.F. (1981). Attention and self-regulation. New York: SpringerVerlag.

Carver, C. S., Ganellen, R. J., Froming, W. J., \& Chambers, W. (1983). Modeling: An analysis in terms of category accessibility. Journal of Experimental Social Psychology, 19, 403-421.

Chen, M., \& Bargh, J.A. (1997). Nonconsciousness behavioral confirmation processes: The self-fulfilling nature of automatically activated stereotypes. Journal of Experimental Social Psychology, 33, 541-560.

Coombes, S.A., Janelle, C.M., \& Duley, A.D. (2005). Emotion and motor control: Movement attributes following affective picture processing. Journal of Motor Behavior, 37, 425-436.

Cox, R.H., Martens, M.P., \& Russell, W.D. (2003). Measuring anxiety in athletics: The revised Competitive State Anxiety Inventory-2. Journal of Sport \& Exercise Psychology, 25, 519-533.

Ford, P., Hodges, N.J., \& Williams, M.A. (2005). Online attentional-focus manipulations in a soccer-dribbling task: Implications for the procedularization of motor skills. Journal of Motor Behavior, 37, 386-394.

Fitts, P.M., \& Posner, M.I. (1967). Human performance. Belmont, CA: Brooks-Cole.

Gray, R. (2004). Attending to the execution of a complex sensorimotor skill: Expertise differences, choking, and slumps. Journal of Experimental Psychology. Applied, 10, 42-54.

Hardy, L., Mullen, R., \& Jones, G. (1996). Knowledge and conscious control of motor actions under stress. The British Journal of Psychology, 87, 621-636.

Hull, J.G., Slone, L.B., Meteyer, K.B., \& Matthews, A.R. (2002). The nonconsiousness of consciousness. Journal of Personality and Social Psychology, 83, 406-424.

Jackson, R.C., Ashford, K.J., \& Norsworthy, G. (2006). Attentional focus, dispositional reinvestment and skilled motor performance under pressure. Journal of Sport \& Exercise Psychology, 28, 49-68.

Lewis, B.P., \& Linder, D.E. (1997). Thinking about choking? Attentional processes and paradoxical performance. Personality and Social Psychology Bulletin, 23, 937-944.

Liao, C., \& Masters, R.S.W. (2002). Self-focused attention and performance failure under psychological stress. Journal of Sport \& Exercise Psychology, 24, 289-305.

MaCrae, N.C., Bodenhausen, G.V., Milne, A B., Castelli, L., Schloercheidt, A.M., \& Greco, S. (1998). On activating exemplars. Journal of Experimental Social Psychology, 34, 330-354.

Masters, R.S.W. (1992). Knowledge, knerves and know-how: The role of explicit versus implicit knowledge in the breakdown of a complex motor skill under pressure. The British Journal of Psychology, 83, 343-358.

Maxwell, J.P., Masters, R.S.W., \& Eves, F.F. (2000). From novice to know-how: A longitudinal study of implicit motor learning. Journal of Sports Sciences, 18, 111-120.

Mullen, R., \& Hardy, L. (2000). State anxiety and motor performance. Testing the conscious processing hypothesis. Journal of Sports Sciences, 18, 785-799.

Nelson, L.D., \& Norton, M.I. (2005). From student to superhero: Situational primes shape future helping. Journal of Experimental Social Psychology, 41, 423-430.

Perkins-Ceccato, N., Passmore, S.R., \& Lee, T.D. (2003). Effect of focus of attention on golfers' skill. Journal of Sports Sciences, 21, 593-600.

Rasinski, K.A., Visser, P.S., Zagatsky, M., \& Rickett, E.M. (2005). Using implicit goal priming to improve the quality of self-report data. Journal of Experimental Social Psychology, 41, 321-327.

Srull, T.K., \& Wyer, R.S. (1979). The role of category accessibility in the interpretation of information about persons: Some determinants and implications. Journal of Personality and Social Psychology, 37, 1660-1672. 
Seinela, A., Hamalainen, P., Kovisto, M., \& Ruutiainen, J. (2002). Conscious and unconscious uses of memory in multiple sclerosis. Journal of the Neurological Sciences, 198, 79-85.

Stone, J., Lynch, C.I., Sjomeling, M., \& Darley, J.M. (1999). Stereotype threat effects on black and white athletic performance. Journal of Personality and Social Psychology, 77, 1213-1227.

Tipper, S.P., \& Weaver, B. (1998). The medium of attention: Location based, object centred, or scene based. In R.D. Wright (Ed.), Visual attention: Vancouver studies in cognitive science (Vol. 8, pp. 77-107). Oxford: Oxford University Press.

Wulf, G., McNevin, N.H., \& Shea, C.H. (2001). The automaticity of complex motor skill learning as a function of attentional focus. The Quarterly Journal of Experimental Psychology, 54A, 1143-1154.

Wulf, G., \& Prinz, W. (2001). Directing attention to movement effects enhances learning. A review. Psychonomic Bulletin \& Review, 8, 648-660.

Manuscript received: August 12, 2009

Revision accepted: April 20, 2010 